\title{
NOUVELLE
}

\section{Des horloges du matin et du soir dans le cerveau de la drosophile}

François Rouyer
Institut de Neurobiologie

Alfred Fessard, CNRS

UPR 2216, Neurobiologie

génétique et intégrative,

avenue de la Terrasse,

91198 Gif-sur-Yvette Cedex,

France.

rouyer@iaf.cnrs-gif.fr

\section{Les saisons et les jours}

Des horloges circadiennes sont présentes dans l'ensemble du monde vivant et contrôlent les rythmes de nombreuses fonctions physiologiques et comportementales, telles que température corporelle ou cycles veillesommeil [1]. Leur période, mesurée en conditions d'obscurité constante, est déterminée génétiquement et d'envi- ron $24 \mathrm{~h}$. En conditions naturelles, l'horloge contrôle la distribution de l'activité au cours du cycle jour-nuit, distribution qui s'avère bimodale chez de nombreux animaux. L'activité est ainsi concentrée à l'aube et au crépuscule, quand les conditions de température et d'humidité sont optimales, en particulier pour les espèces qui ne régulent
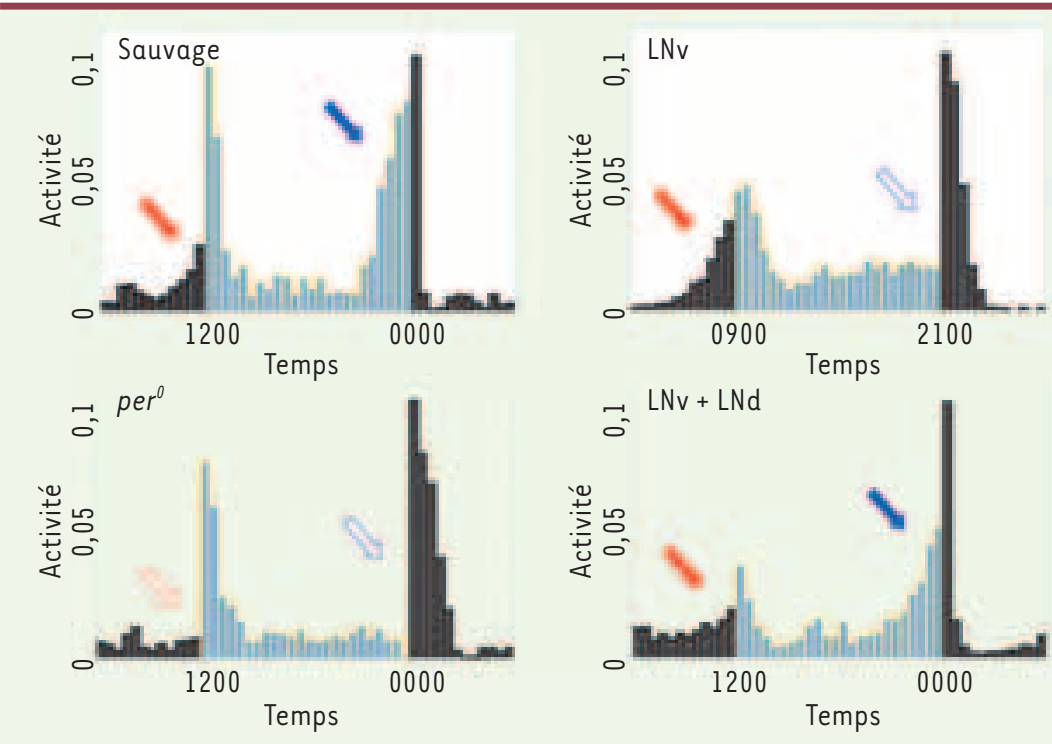

Figure 1. Rythmes d'activité locomotrice en conditions jour-nuit. Les 4 quatre graphes représentent la distribution moyenne de l'activité locomotrice au cours d'un cycle jour-nuit de $24 \mathrm{~h}$. Les flèches indiquent la présence (flèche pleine) ou l'absence (flèche vide) d'une anticipation des transitions nuit-jour (rouge) ou jour-nuit (bleu). Cette anticipation atteste de l'existence d'un contrôle circadien de l'activité du matin ou du soir. Chez une mouche sauvage, les deux pics circadiens sont présents. Chez le mutant per $^{0}$, les deux anticipations sont absentes, mais les mouches gardent une réponse non circadienne à l'allumage et à l'extinction de la lumière. Les mouches n'exprimant PER que dans les LNv ont le seul pic d'activité du matin, tandis que celles exprimant $P \varepsilon R$ dans les $L N v$ et les $L N d$ ont les deux pics.

pas leur température interne. Lorsque la durée du jour (photopériode) change en fonction de la saison, les pics d'activité se déplacent et restent ainsi calés sur l'aube et le crépuscule de la nouvelle photopériode. L'adaptation saisonnière implique donc une réponse opposée des pics du matin et du soir à la lumière, suggérant que deux oscillateurs différents contrôlent les rythmes veille-sommeil [2]. Cette hypothèse est étayée par le phénomène de splitting observé chez différents rongeurs, lorsqu'ils sont placés en conditions de lumière constante ( $L L)$. Après quelques jours dans ces conditions, certains animaux montrent alors une séparation du rythme d'activité en deux composants: I'un à période courte et l'autre à période longue. C. Pittendrigh et S.A. Daan [2] ont proposé que ces composants reflètent l'existence de deux oscillateurs, un oscillateur matinal accéléré par la lumière, et un oscillateur vespéral ralenti par la lumière.

Les horloges des jours chez la drosophile

Deux études ont récemment mis en évidence les bases neuronales de ces oscillateurs du matin et du soir chez la drosophile $[3,4]$.

L'horloge qui gouverne le rythme d'activité locomotrice est située dans le cerveau, où les quantités des produits des gènes period (per) et timeless (tim) oscillent dans une centaine de neurones par hémisphère cérébral répartis en plusieurs groupes dorsaux et latéraux [5]. Un sous-groupe ventral de neurones latéraux (LNv) exprime le neuropeptide PDF (pigment-dispersing factor), dont l'absence induit une arythmicité 
importante en conditions d'obscurité constante [6]. Afin de caractériser la contribution des différents groupes de neurones d'horloge à la rythmicité comportementale, les deux laboratoires ont utilisé le système Gal4/UAS qui permet de cibler l'expression de gènes dans certains groupes de cellules pour lesquels on dispose d'une séquence stimulatrice transcriptionnelle spécifique (enhancer). $\varepsilon$ n bref, un transgène portant le facteur de transcription de levure Gal4 sous le contrôle d'un enhancer tissu-spécifique va diriger l'expression d'un gène d'intérêt placé derrière les séquences UAS de réponse à Gal4 dans un second transgène. Il avait été montré précédemment que l'expression d'un gène d'apoptose sous le contrôle de transgènes Gal4 spécifiquement exprimés dans les neurones à PDF provoque une ablation génétique de ces neurones et induit des défauts similaires à ceux observés chez les mutants pdfo $[6,7]$. Les neurones latéro-ventraux à
PDF étaient donc de bons candidats pour être le siège de l'oscillateur principal contrôlant les rythmes veille-sommeil de la drosophile.

\section{Bases neuronales de l'oscillateur} du matin

En ciblant l'expression du gène per dans ces neurones chez des mouches dépourvues de gène per endogène (mutants per0), B. Grima et al. [3] montrent que les LNv sont capables à eux seuls d'engendrer une rythmicité comportementale de 24 h. Les LNv à PDF (une dizaine de cellules par hémisphère) constituent donc une horloge circadienne autonome. Ces mouches présentent cependant une avance de phase de plusieurs heures, la phase du rythme représentant la position du pic d'activité dans un cycle de $24 \mathrm{~h}$. $\varepsilon n$ conditions jour-nuit, les mouches n'exprimant la protéine PER que dans les LNv ne possèdent que le pic du matin (Figure 1), tandis que les mutants pdfo n'ont au contraire que le pic du soir. En induisant l'ablation spécifique des LNv, D. Stoleru et al. [4] obtiennent également la perte du pic du matin. II apparaît donc que les LNv à PDF sont responsables du contrôle circadien de l'activité matinale.

Bases neuronales de l'oscillateur du soir

Quels sont les neurones responsables de l'activité du soir? B. Grima et al. ont utilisé un autre transgène Gal4 qui induit l'expression de PER dans les LNv à PDF ainsi que dans une partie des neurones latéro-dorsaux ( $L N d)$, situés de façon plus dorsale et n'exprimant pas le PDF. Les mouches produites retrouvent alors un pic du matin et un pic du soir [3] (Figure 1). D. Stoleru et al. montrent que l'ablation génétique des neurones d'horloge, à l'exception des LNv à PDF, abolit spécifiquement l'activité du soir [4]. Cette ablation sélective des neurones
Une synthèse unique sur les mécanismes de la mémoire humaine et animale qui s'appuie aussi bien sur les travaux utilisant l'imagerie fonctionnelle que sur les études psychologiques et neurophysiologiques d'animaux et de patients atteints de troubles de la mémoire.

Larry Squire est professeur de poychiatrie, neurnociences et puschologie â Tuniversité de San Diego.

firis Kandel, professeru à Tunivenite Contanbia a New-york, a resu le prix Nobel de physiologie et de médecine en 2000.

\section{SQUIRE • KANDEL} LA MÉMOIRE.

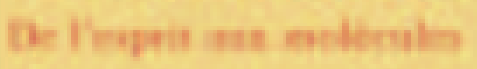

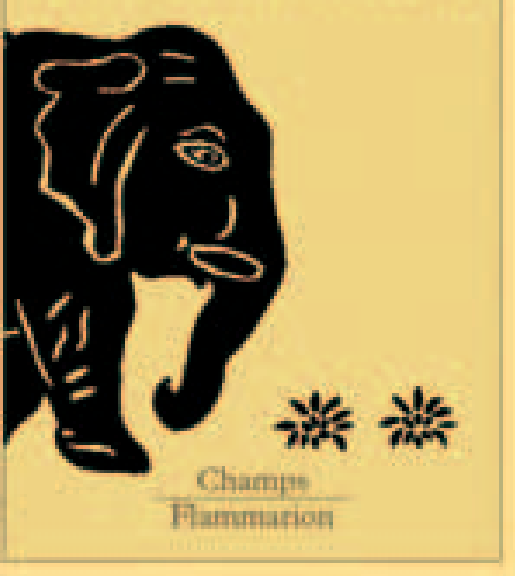


PDF négatifs est élégamment obtenue par l'expression d'un gène d'apoptose sous le contrôle d'un Gal4 exprimé dans l'ensemble des neurones d'horloge, couplée à l'inhibition du Gal4 par son inhibiteur Gal80 dans les seuls neurones à PDF. Les résultats de ces deux études démontrent la présence de deux oscillateurs dans le cerveau de la mouche, l'un porté par les LNv à PDF qui contrôle l'activité du matin (M), l'autre porté par les LNd PDF-négatifs qui contrôle l'activité du soir ( $\varepsilon$ ) (Figure 2). Contrairement à celui du matin, l'oscillateur du soir porté par les LNd semble ne fonctionner qu'en conditions LD (alternance jour-nuit), mais participe pourtant à la production du rythme comportemental en DD (obscurité constante) en présence des LNv. En effet, l'avance de phase observée en obscurité constante, chez les mouches n'ayant que l'oscillateur du matin, est perdue chez celles qui expriment $P E R$ dans les deux oscillateurs, qui se rapprochent des mouches sauvages.

\section{Vers la complexité de l'horloge \\ des mammifères}

L'architecture neuronale de l'horloge est cependant loin d'avoir livré tous ses secrets puisque le gène per est également exprimé dans trois groupes de neurones dorsaux dont on ignore la fonction, à l'exception de leur participation à la synchronisation de l'horloge par la lumière. Des analyses comportementales réalisées en LL (lumière constante) montrent que le phénomène de splitting existe également chez la drosophile et suggèrent que ces groupes dorsaux participent aux composants matinaux et vespéraux de la rythmicité circadienne [8]. Les interactions fonctionnelles entre les oscillateurs des différents groupes neuronaux restent à définir, ainsi que leur rôle dans l'entrầnement de l'horloge par les cycles journuit et son adaptation aux variations saisonnières de la photopériode. Néanmoins, une porte a été ouverte sur le fonctionnement du réseau de neurones de l'horloge cérébrale. Chez les rongeurs, des oscillations de l'activité électrophysiologique du noyau suprachiasmatique (SCN) comportant une composante du matin et une composante du soir ont été enregistrées in vitro [9]. L’horloge qui gouverne les rythmes veille-sommeil des mammifères est située dans les noyaux suprachiasmatiques, constitués

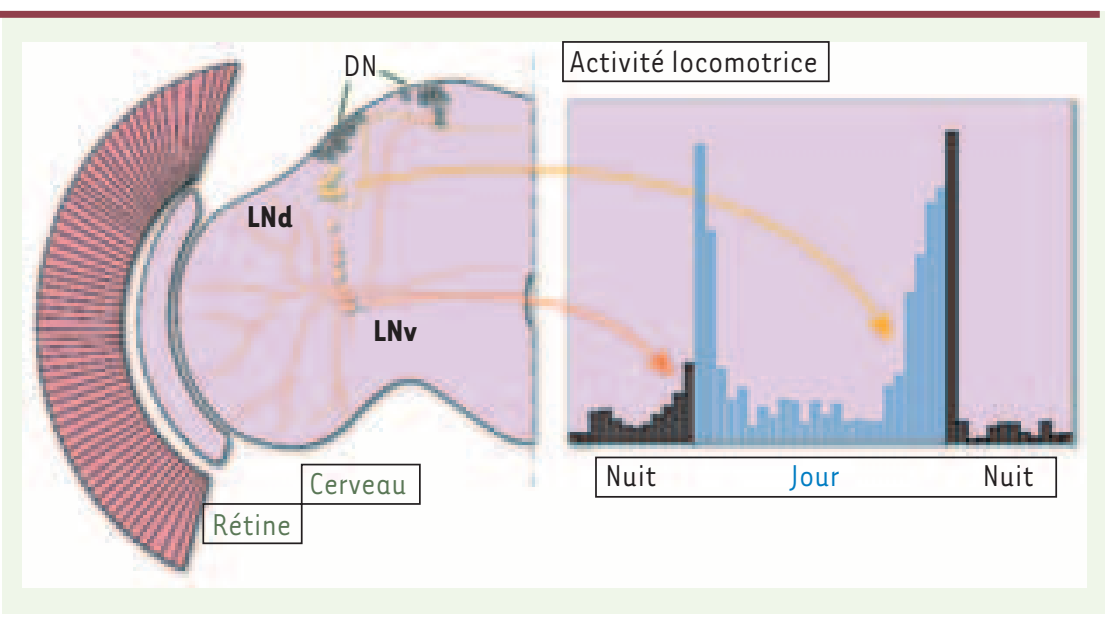

Figure 2. Contrôle des activités du matin et du soir par deux groupes de neurones latéraux différents. Un hémisphère cérébral de drosophile contient une centaine de neurones d'horloge exprimant la protéine PERIOD et répartis en groupes latéraux (LN) et dorsaux (DN). Les neurones latéraux ventraux (LNv) contrôlent l'activité circadienne du matin, tandis que les neurones latéraux dorsaux (LNd) contrôlent l'activité circadienne du soir. d'environ 10000 neurones. L'interprétation des données électrophysiologiques en termes neuronaux est confrontée à la complexité de l'organisation cellulaire des SCN, mais les travaux réalisés ces dernières années indiquent clairement qu'ils sont composés de différents oscillateurs [10]. Couplées aux avancées considérables réalisées sur les aspects moléculaires du fonctionnement des oscillateurs circadiens [11], ces études nous permettront peut-être de comprendre bientôt pourquoi certains d'entre nous sont plutôt du soir ou plutôt du matin. $\diamond$

Morning and evening clocks in the fly brain

\section{RÉFÉRENCES}

1. Dunlap J, Loros J, DeCoursey PJ. Chronobiology. Biological timekeeping. Sunderland, MA, USA : Sinauer Associates, 2004.

2. Pittendrigh C, Daan SA. Functional analysis of circadian pacemakers in nocturnal rodents. $V$. Pacemaker structure: a clock for all seasons. J Comp Physiol 1976; Al06: 333-5.

3. Grima B, Chélot $\varepsilon$, Xia R, Rouyer F. Morning and evening peaks of activity rely on different clock neurons of the Drosophila brain. Nature 2004 ; 431 : 869-73.

4. Stoleru D, Peng P, Agosto J, Rosbash M. Coupled oscillators control morning and evening locomotor behavior of Drosophila. Nature 2004 ; $431: 862-8$.

5. Hall JC. Genetics and molecular biology of rhythms in Drosophila and other insects. Adv Genet 2000 ; 348 : 1-28.

6. Renn SC, Park JH, Rosbash M, et al. A pdf neuropeptide gene mutation and ablation of PDF neurons each cause severe abnormalities of behavioral circadian rhythms in Drosophila. Cell 1999; $99: 791-802$.

7. Blanchardon $\varepsilon$, Grima B, Klarsfeld A, et al. Defining the role of Drosophila lateral neurons in the control of circadian activity and eclosion rhythms by targeted genetic ablation and PERIOD protein overexpression. Eur J Neurosci 2001 ; 13 : 871-88.

8. Yoshii T, Funada Y, Ibuki-Ishibashi T, et al. Drosophila cry (b) mutation reveals two circadian clocks that drive locomotor rhythm and have different responsiveness to light. J Insect Physiol 2004 ; 50 : 479 88.

9. Jagota, A, De la Iglesia HO, Schwartz WJ. Morning and evening circadian oscillations in the suprachiasmatic nucleus in vitro. Nat Neurosci $2000 ; 3: 372-6$.

10. Antle MC, Silver R. Orchestrating time: arrangements of the brain circadian clock. Trends Neurosci 2005 ; 28 : 145-51.

11. Bell-Pedersen D, Cassone VM, Earnest DJ, et al. Circadian rhythms from multiple oscillators: lessons from diverse organisms. Nat Rev Genet 2005 ; 6 : 544 56. 